\title{
Understanding Lactatemia in Human Sepsis Potential Impact for Early Management
}

\begin{abstract}
Luciano Gattinoni ${ }^{1}$, Francesco Vasques ${ }^{2,3}$, Luigi Camporota ${ }^{2,3}$, Jennifer Meessen ${ }^{4}$, Federica Romitti ${ }^{1}$, lacopo Pasticci ${ }^{1}$, Eleonora Duscio ${ }^{1}$, Francesco Vassalli ${ }^{1}$, Lui G. Forni ${ }^{5,6}$, Didier Payen ${ }^{7}$, Massimo Cressoni ${ }^{8}$, Alberto Zanella ${ }^{9,10}$, Roberto Latini ${ }^{4}$, Michael Quintel ${ }^{1}$, and John J. Marini ${ }^{11,12}$

${ }^{1}$ Department of Anaesthesiology, Emergency and Intensive Care Medicine, University of Göttingen, Göttingen, Germany; ${ }^{2}$ Department of Adult Critical Care, Guy's and St. Thomas' NHS Foundation Trust, London, United Kingdom; 'Health Centre for Human and Applied Physiological Sciences, King's College London, London, United Kingdom; ${ }^{4}$ Dipartimento di Ricerche Cardiovascolari, Istituto di Ricovero e Cura a Carattere Scientifico Istituto di Ricerche Farmacologiche "Mario Negri," Milan, Italy; ${ }^{5}$ Department of Intensive Care Medicine, Royal Surrey County Hospital NHS Foundation Trust, Guildford, United Kingdom; ${ }^{6}$ Faculty of Health Sciences, University of Surrey, Guildford, United Kingdom; 'Department of Anaesthesiology and Critical Care, Lariboisière University Hospital, Public Assistance-Paris Hospital, University Paris Diderot, Paris, France; ${ }^{8}$ Dipartimento di Scienze della Salute, Università degli Studi di Milano Bicocca, Milan, Italy; ${ }^{9}$ Anaesthesia and Critical Care, Department of Pathophysiology and Transplantation, University of Milan, Milan, Italy; ${ }^{10}$ Dipartimento di Anestesia, Rianimazione ed Emergenza, Fondazione Istituto di Ricerca e Cura a Carattere Scientifico Ca' Granda Ospedale Maggiore Policlinico, Milan, Italy; ${ }^{11}$ Regions Hospital, St. Paul, Minnesota; and ${ }^{12}$ University of Minnesota, St. Paul, Minnesota
\end{abstract}

ORCID ID: 0000-0001-5380-2494 (L.G.).

\section{Abstract}

Rationale: Hyperlactatemia in sepsis may derive from a prevalent impairment of oxygen supply/demand and/or oxygen use.

Discriminating between these two mechanisms may be relevant for the early fluid resuscitation strategy.

Objectives: To understand the relationship among central venous oxygen saturation $\left(\mathrm{Scv}_{\mathrm{O}_{2}}\right)$, lactate, and base excess to better determine the origin of lactate.

Methods: This was a post hoc analysis of baseline variables of 1,741 patients with sepsis enrolled in the multicenter trial ALBIOS (Albumin Italian Outcome Sepsis). Variables were analyzed as a function of sextiles of lactate concentration and sextiles of $\mathrm{Scv}_{\mathrm{O}_{2}}$. We defined the "alactic base excess," as the sum of lactate and standard base excess.

Measurements and Main Results: Organ dysfunction severity scores, physiologic variables of hepatic, metabolic, cardiac, and renal function, and 90-day mortality were measured. $\mathrm{Scv}_{\mathrm{O}_{2}}$ was lower than $70 \%$ only in $35 \%$ of patients. Mortality, organ dysfunction scores, and lactate were highest in the first and sixth sextiles of $\mathrm{Scv}_{\mathrm{O}_{2}}$. Although lactate level related strongly to mortality, it was associated with acidemia only when kidney function was impaired (creatinine $>2 \mathrm{mg} / \mathrm{dl}$ ), as rapidly detected by a negative alactic base excess. In contrast, positive values of alactic base excess were associated with a relative reduction of fluid balance.

Conclusions: Hyperlactatemia is powerfully correlated with severity of sepsis and, in established sepsis, is caused more frequently by impaired tissue oxygen use, rather than by impaired oxygen transport. Concomitant acidemia was only observed in the presence of renal dysfunction, as rapidly detected by alactic base excess. The current strategy of fluid resuscitation could be modified according to the origin of excess lactate.

Keywords: sepsis; lactic acidosis; venous oxygen saturation; base excess

(Received in original form December 17, 2018; accepted in final form April 11, 2019)

Supported by a generous donation from Ilse Liselotte Munz to the Department of Anesthesiology of Göttingen.

Author Contributions: Conception and design: L.G., M.Q., and J.J.M. Data collection: I.P., E.D., M.C., and A.Z. Analysis and interpretation: F. Vasques, J.M., F.R., L.G.F., D.P., and R.L. Drafting the manuscript for important intellectual content: L.G., F. Vasques, L.C., F. Vassalli, and J.J.M.

Correspondence and requests for reprints should be addressed to Luciano Gattinoni, M.D., Department of Anaesthesiology, Emergency and Intensive Care Medicine, University of Göttingen, Robert-Koch-Straße 40, 37075 Göttingen, Germany. E-mail: gattinoniluciano@gmail.com.

This article has an online supplement, which is accessible from this issue's table of contents at www.atsjournals.org.

Am J Respir Crit Care Med Vol 200, Iss 5, pp 582-589, Sep 1, 2019

Copyright $\odot 2019$ by the American Thoracic Society

Originally Published in Press as DOI: 10.1164/rccm.201812-2342OC on April 15, 2019

Internet address: www.atsjournals.org 


\section{At a Glance Commentary}

\section{Scientific Knowledge on the}

Subject: Hyperlactatemia may originate from different causes and in patients with sepsis may occur regardless of tissue oxygenation. Indeed, hyperlactatemia may occur in the presence of high or low values of central venous oxygen saturation. In addition, hyperlactatemia may occur with or without acidemia.

\section{What This Study Adds to the}

Field: Using a large database of patients with sepsis, we validated previous discussions on lactate. Data suggest that impairment of oxygen use occurs more frequently than impairment of oxygen transport. Acidemia is associated with hyperlactatemia primarily when renal function is impaired. A negative alactic base excess, which estimates negative strong ions other than lactate, immediately suggests the presence of renal dysfunction.

Hyperlactatemia has historically been associated with adverse outcomes in critically ill patients (1) and still represents the strongest outcome indicator in sepsis. Hyperlactatemia, however, may originate from a variety of causes, such as a deficit in oxygen delivery (tissue hypoxia) or impaired oxygen extraction (2), peripheral shunting (3), stress (4), and increased adrenergic stimulation (5). It is not clear, however, in what proportions these different events may occur in patients with sepsis, and how their relative importance could be clinically discriminated.

Furthermore, hyperlactatemia may occur in presence or absence of acidemia, and the reasons for this variability are still unclear. Finally, but importantly, we wonder if better recognition of the mechanism of hyperlactatemia and acidemia would influence the treatment of patients with sepsis and positively alter their outcome.

To address these questions, we propose a unifying interpretation of the pathophysiology of lactate in sepsis. This approach broadens one previously suggested $(6,7)$, and still accepted by many: lactate elevation arises from tissue hypoxia that originates primarily from a deficit in oxygen transport. This common clinical perception often motivates adherence to the current resuscitation approach of aggressive and indiscriminate nonselective administration of fluid. Our expanded interpretation is based on accepted principles that are well known and documented in isolation but have never been grouped together and presented as a unified model. We applied this unifying conceptual interpretation to a large dataset of patients with sepsis, derived from the ALBIOS (Albumin Italian Outcome Sepsis) study (8), using the baseline data collected before randomization. We hypothesized that, after accounting for potentially relevant confounders, hyperlactatemia is present both at high and low values of central venous oxygen saturation $\left(\mathrm{Scv}_{\mathrm{O}_{2}}\right)$ and that the presence or absence of kidney injury determines the final effect of plasma lactate concentration on $\mathrm{pH}$.

\section{Methods}

\section{Patients}

This study is a secondary analysis of the ALBIOS study (8), a multicenter randomized controlled trial conducted between 2008 and 2012 in 100 Italian ICUs that compared the effects of $20 \%$ albumin and crystalloids versus crystalloids alone in severe sepsis and septic shock. In the present study, we included 1,741/1,818 patients for whom both serum lactate and $\mathrm{Scv}_{\mathrm{O}_{2}}$ measurements were available at baseline (see Figure E1 in the online supplement). Measurements were collected at baseline (within $24 \mathrm{~h}$ from the diagnosis of sepsis) after randomization and before the albumin administration. We do not know the volume or composition of fluids given to the patients in the emergency room and/or in ICU before the randomization. Therefore, our analysis refers to the subsequent phase of sepsis management.

\section{Study Design}

We analyzed baseline clinical, physiologic, and hemodynamic variables as functions of lactate concentration, $\mathrm{Scv}_{\mathrm{O}_{2}}$ levels, and alactic base excess (BE) (see below). These variables were grouped into sextiles that included similar numbers of patients $(<250$ each $)$.

\section{Measured Variables}

Clinical. We recorded Sequential Organ Failure Assessment score (SOFA) (9), Simplified Acute Physiology Score II (10), 90-day mortality, bilirubin, glucose, creatinine, albumin, platelet and leukocyte count, percentage of patients fulfilling the Sepsis-2 definition (as defined by the ALBIOS study entry criteria [8]) or Sepsis-3 criteria (i.e., vasopressor requirement to maintain mean arterial pressure $\geqslant 65 \mathrm{~mm} \mathrm{Hg}$ with lactate levels $>2 \mathrm{mmol} / \mathrm{L})(11,12)$, and proportion of patients requiring renal replacement therapy (RRT).

Physiologic. Physiologic measurements included $\mathrm{F}_{\mathrm{O}_{2}}$, arterial and venous partial pressures of oxygen and carbon dioxide, arterial and venous $\mathrm{pH}$, arterial $\mathrm{BE}$, sodium, chloride and potassium, diuresis, and fluid balance in the first 6 hours after admission.

Hemodynamic. Hemodynamic measurements included central venous and mean arterial pressures, heart rate, use and dosing of epinephrine, norepinephrine, $\mathrm{SCV}_{\mathrm{O}_{2}}$, arteriovenous difference in oxygen content, venoarterial difference of $\mathrm{CO}_{2}$ partial pressures, and $\mathrm{Hb}$ concentration.

\section{Computed Variables}

We computed the standard $\mathrm{BE}$ as

$$
\begin{aligned}
& \text { standard } \mathrm{BE}(\mathrm{mmol} / \mathrm{L}) \\
& \quad=\left[\mathrm{HCO}_{3}^{-}(\mathrm{mmol} / \mathrm{L})-24.8 \mathrm{mmol} / \mathrm{L}\right] \\
& \quad+16.2 \mathrm{mmol} / \mathrm{L} \times(\mathrm{pH}-7.4) .
\end{aligned}
$$

We used standard BE rather than actual as better representative of the buffer base status of the extracellular fluid (13). See the online supplement for details.

To better understand the relationship between hyperlactatemia and acidemia, we introduce the concept of alactic BE, which helps in the rapid discrimination between metabolic acidosis secondary to lactate accumulation from that caused by an increase in fixed acids (unmeasured strong anions):

$$
\begin{aligned}
& \text { alactic BE }(\mathrm{mmol} / \mathrm{L}) \\
& =\text { standard base excess }(\mathrm{mmol} / \mathrm{L}) \\
& + \text { lactate }(\mathrm{mmol} / \mathrm{L}) .
\end{aligned}
$$

This variable focuses on the role of fixed acids other than lactate in the sepsis scenario (fixed acids refer to the acids 
[e.g., phosphates and sulfates] that cannot be eliminated through the lungs).

\section{Statistics}

Patient characteristics are reported as mean $\pm \mathrm{SD}$. Lactate and $\mathrm{Scv}_{\mathrm{O}_{2}}$ were divided into sextiles. The division into sextiles was arbitrarily decided to provide reasonable resolution power of the model, while maintaining adequate patient number in each quantile $(\sim 250)$. In this way, the results are more easily understandable than splitting the independent variables according to restricted cubic splines, which provide, however, similar results (see online supplement for details). Comparison of continuous variables among groups was made through one-way ANOVA test with Tukey honest significant difference test for pair-wise comparisons. Dichotomous variables were compared using the chisquare test. A $P$ value less than 0.05 was considered statistically significant. All statistical analyses were performed using $\mathrm{R}$ and GraphPad Prism Software.

\section{Study Approval}

The protocol of the original ALBIOS study and the informed-consent process were approved by the ethics committee at each participating institution. Written informed consent or deferred consent was obtained from each patient.

\section{Results}

\section{Lactate and Clinical Variables}

As shown in Figure 1, mortality and SOFA score progressively increased across the sextiles of lactate. In contrast, the $\mathrm{Scv}_{\mathrm{O}_{2}}$ remained remarkably similar $\left(\mathrm{Scv}_{\mathrm{O}_{2}} \sim 72 \%\right.$ ) throughout the first five sextiles of lactate (i.e., lactate levels ranging from 0.1 to 5.6 $\mathrm{mmol} / \mathrm{L}$ ) and slightly, but significantly, decreased to $69.7 \%$ in the highest lactate sextile (i.e., lactate levels from 5.6 to 27 $\mathrm{mmol} / \mathrm{L})$. Similarly, $\mathrm{pH}$ remained similar and in the normal range within the first five sextiles of lactate and decreased significantly to a mean \pm SD of $7.31 \pm 0.14$ only in the terminal lactate sextile. Most of the other measured clinical, physiologic, and hemodynamic variables deteriorated with increasing lactate levels, as reported in Table E1. Briefly, central venous pressure $(P=0.01)$, heart rate $(P<0.001)$, norepinephrine requirements, mean arterial pressure $(P<0.001)$, diuresis, and fluid balance deteriorated progressively $(P<0.001) ; \mathrm{Pa}_{\mathrm{O}_{2}}$ and $\mathrm{Pa}_{\mathrm{CO}_{2}}$ values were similar across all lactate sextiles. There was no obvious relationship between alactic $\mathrm{BE}$ and lactate level.

\section{Scv $_{\mathrm{O}_{2}}$, Lactate, and Tissue Hypoxia}

As shown in Figure 2, the $\mathrm{Scv}_{\mathrm{O}_{2}}$, as recorded in all the patients with sepsis without exclusions, ranged from $24 \%$ to $98 \%$ (median, 73\%; interquartile range, 67-79\%). As shown in Figure 3, several clinical variables, including lactate, SOFA score, and mortality, showed a U-shaped relationship with the $\mathrm{Scv}_{\mathrm{O}_{2}}$. Indeed, the worst values of these variables were observed at the lowest and highest levels of $\mathrm{Scv}_{\mathrm{O}_{2}}$. Among the other clinical, hemodynamic, and laboratory variables grouped by sextiles of increasing $\mathrm{Scv}_{\mathrm{O}_{2}}$ (see Table E2), creatinine and RRT displayed $\mathrm{U}$-shaped behavior, central venous oxygen pressure, $\mathrm{Pa}_{\mathrm{O}_{2}}, \mathrm{~Pa}_{\mathrm{CO}_{2}}$, and central venous carbon dioxide pressure progressively increased $(P<0.001)$; $\mathrm{pH}(P<0.003)$ and $\mathrm{BE}(P<0.001)$ significantly decreased, whereas the arteriovenous difference in oxygen content showed a threefold decrease (from 6 to $2 \mathrm{ml} / \mathrm{dl}$ ) from the first to the sixth $\mathrm{Scv}_{\mathrm{O}_{2}}$ sextile $(P<0.001)$. Conversely, central venous pressure, heart rate, and vasoactive drugs requirements were similar for all sextiles of $\mathrm{Scv}_{\mathrm{O}_{2}}$. The average value of mean arterial pressure remained above $70 \mathrm{~mm} \mathrm{Hg}$, showing no clear relationship with the $\mathrm{Scv}_{\mathrm{O}_{2}}$ level.

\section{Lactate, Acidosis, and Alactic BE}

According to the physicochemical approach of Stewart to the acid-base equilibrium (14), an increase in lactate (a strong negative ion) leads to a decrease in the strong ion difference, which finally results in metabolic acidosis and acidemia. Therefore, if the $\mathrm{pH}$ is not corrected by compensatory mechanisms, the lactate per se always produces acidemia. Actually, out of 1,017 patients with lactate greater than $2 \mathrm{mmol} / \mathrm{L}$, $57 \%$ had normal pH $(>7.35)$ (Figure 1D; see Table E1). To better understand this lack of a consistent correlation between hyperlactatemia and acidemia, we introduce the concept of alactic BE. This variable equals the amount of strong acids, other than lactate, which are present in the plasma in abnormal concentrations. The alactic BE was related to kidney function (Figure 4), as indicated by its relationship to creatinine levels, urine output, and use of
RRT. Accordingly, with worsening renal function, the concentration of fixed acids other than lactate increased in the plasma. This dysfunction led to worsening acidemia, as reflected in more negative values of alactic BE. Conversely, an alactic $\mathrm{BE}$ near 0 suggested that acidemia was fully explained by the lactate, because no other acids were present in excess, whereas a positive alactic BE suggested either that the kidney fully compensated for metabolic acidosis or that additional mechanisms contributed to metabolic alkalosis (e.g., diuretic usage, contraction of the extracellular volume). Actually, the alactic $\mathrm{BE}$ was strongly associated to the fluid balance (see Figure E5). In Table E3, we summarize the most relevant clinical and physiologic variables as functions of sextiles of alactic BE.

\section{A Comprehensive Synthesis of the Results}

In Figure 5 we present an integrated view of our results. As shown, hyperlactatemia was increased quite independently from $\dot{\mathrm{V}}_{\mathrm{O}_{2}}$ / oxygen delivery $\left(\mathrm{D}_{\mathrm{O}_{2}}\right.$ ) (see Figure E6A). In contrast the $\dot{\mathrm{V}}_{\mathrm{O}_{2}} / \mathrm{D}_{\mathrm{O}_{2}}$, viewed as an independent variable, strictly determines the $\mathrm{Scv}_{\mathrm{O}_{2}}$ levels: low when the oxygen transport is low (high $\dot{\mathrm{V}}_{\mathrm{O}_{2}} / \mathrm{D}_{\mathrm{O}_{2}}$ ) and high when oxygen use is impaired (low $\dot{\mathrm{V}}_{\mathrm{O}_{2}} / \mathrm{D}_{\mathrm{O}_{2}}$ ). The physiologically sound $\mathrm{Scv}_{\mathrm{O}_{2}}-\mathrm{V}_{\mathrm{O}_{2}} / \mathrm{D}_{\mathrm{O}_{2}}$ relationship, unfortunately, is biased by mathematical coupling, which prevents a rigorous analysis of possible confounders. In addition, it is worth emphasizing that $\mathrm{Scv}_{\mathrm{O}_{2}}$ might not be representative of the wholebody average oxygen venous saturation, even though it is a broadly accepted surrogate of the mixed venous oxygen saturation (15). The second independent variable is renal function, on which we hypothesize that acidemia should primarily depend. Among several variables, we found that $\mathrm{PC}_{\mathrm{O}_{2}}$, SOFA without its renal component, and mean arterial pressure acted as real confounders both on creatinine and $\mathrm{pH}$. Including these variables in a multiple linear regression model, the creatinine remained the variable most strongly independently related to the $\mathrm{pH}$ (see online supplement for complete analysis).

\section{Discussion}

Over recent decades there has been a growing evidence that lactate in sepsis and 
A

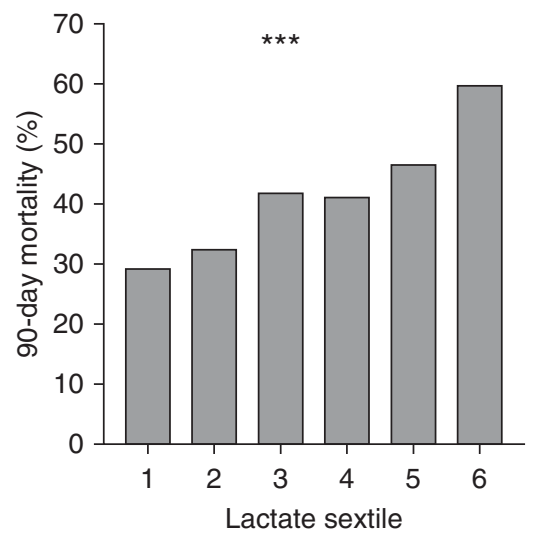

C

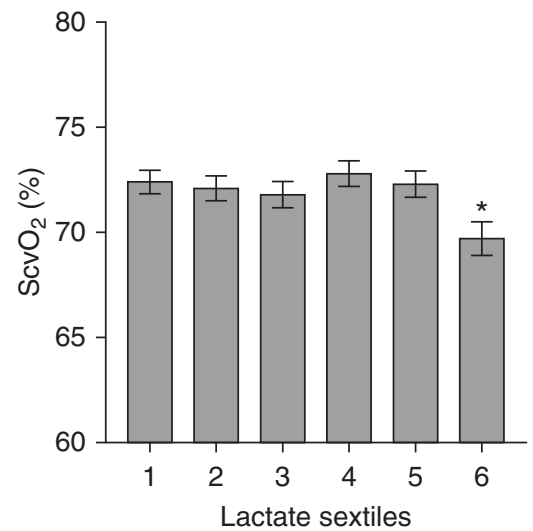

B

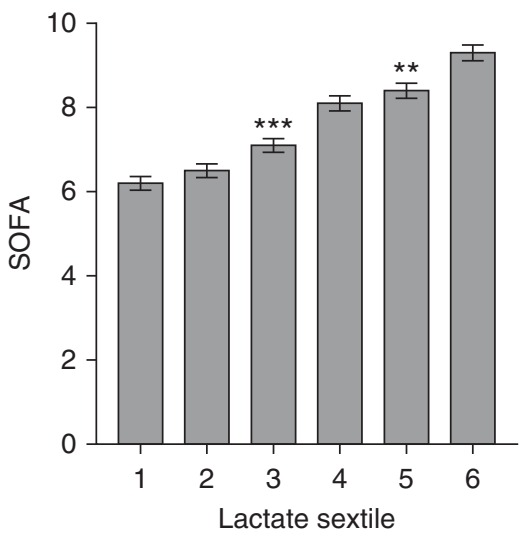

D

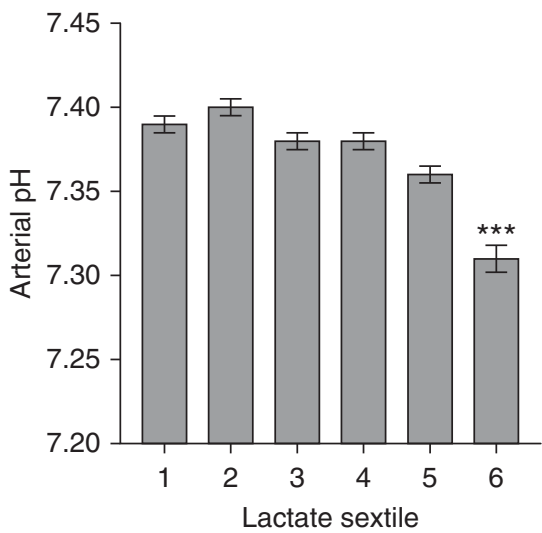

Figure 1. $(A-D)$ The 90-day mortality $(A)$, Sequential Organ Failure Assessment score $(B)$, central venous oxygen saturation $(C)$, and arterial $\mathrm{pH}(D)$ as a function of lactate sextiles at baseline (ICU admission). Data are presented as mean $\pm S E$. Lactate sextile ranges: $1,0.1-1.2 \mathrm{mmol} \cdot \mathrm{L}^{-1} ; 2$, $1.2-1.8 \mathrm{mmol} \cdot \mathrm{L}^{-1} ; 3,1.8-2.5 \mathrm{mmol} \cdot \mathrm{L}^{-1} ; 4,2.5-3.5 \mathrm{mmol} \cdot \mathrm{L}^{-1} ; 5,3.5-5.6 \mathrm{mmol} \cdot \mathrm{L}^{-1}$; and 6 , 5.6-27 mmol $\cdot L^{-1}$. Level of statistical significance: ${ }^{\star} P<0.05$, ${ }^{\star \star} P<0.01$, and ${ }^{\star \star \star}{ }^{\star} P<0.001$. The level of significance represented in $A$ refers to the chi-square test, whereas for $B-D$ it refers to pairwise comparison in ANOVA model. Only significant comparisons are displayed. $\mathrm{ScV}_{\mathrm{O}_{2}}=$ central venous oxygen saturation; SOFA = Sequential Organ Failure Assessment score.

shock state may increase for several reasons other than tissue hypoxia (16). The possible heterogenous sources of lactate in septic shock, however, have rarely been quantified. Alegría and colleagues (17) in a retrospective analysis of 90 patients with septic shock, found that 70 patients presented elevated lactate in association with signs of hypoperfusion (including $\mathrm{Scv}_{\mathrm{O}_{2}}<70 \%$ ). In our analysis on 1,741 patients, after admission in ICU, we found that only $35 \%$ of the patients had an $\mathrm{Scv}_{\mathrm{O}_{2}}$ less than $70 \%$, whereas $65 \%$ had high lactate coexisting with normal or increased $\mathrm{Scv}_{\mathrm{O}_{2}}$. This finding suggests that high lactate levels, as observed in an ICU setting after initial fluid resuscitation made in the emergency department, are caused by a macrocirculatory oxygen transport defect only in a minority of cases. Furthermore, we found that hyperlactatemia in this setting is reliably associated with acidemia only if renal dysfunction is simultaneously present. Finally, the estimation of the alactic BE is a useful tool by which the degree of renal compensation of the acidbase disorder can be rapidly determined.

\section{Lactate and Tissue Hypoxia}

Despite its limitations, $\mathrm{Scv}_{\mathrm{O}_{2}}$ is one of the best surrogates for the assessment of tissue oxygen availability (i.e., the relationship between oxygen delivery and demand) and is widely used in clinical practice. We found that, on admission, only approximately $35 \%$ of our patients had $\mathrm{Scv}_{\mathrm{O}_{2}}$ lower than
$70 \%$. This finding is consistent with what has been observed in most large clinical trials performed for sepsis (18-20). Although, admittedly, $\mathrm{Scv}_{\mathrm{O}_{2}}$ is an imperfect indicator of the cellular oxygen environment, it is reasonable to associate extreme values of $\mathrm{Scv}_{\mathrm{O}_{2}}$ either to a predominant oxygen transport insufficiency (low $\mathrm{Scv}_{\mathrm{O}_{2}}$ ) or to a predominant oxygen use impairment (high $\mathrm{Scv}_{\mathrm{O}_{2}}$ ). These two extremes of $\mathrm{Scv}_{\mathrm{O}_{2}}$ are indeed associated with the highest lactate levels, renal dysfunction, disease severity, and mortality, so that $\mathrm{Scv}_{\mathrm{O}_{2}}$ has a U-shaped relationship with these characteristics. This interpretation is supported by other findings: the highest arteriovenous oxygen content difference and the greatest venoarterial difference in $\mathrm{PC}_{\mathrm{O}_{2}}$ were found in the first $\mathrm{ScV}_{\mathrm{O}_{2}}$ sextile (24-62\%).

At the opposite extreme, the presence of hyperlactatemia at the most elevated $\mathrm{Scv}_{\mathrm{O}_{2}}$ levels (78-98\%) strongly suggests mechanisms other than an oxygen transport deficit. In sepsis, elevated lactate levels with high $\mathrm{Scv}_{\mathrm{O}_{2}}$ may be explained by a variety of mechanisms ranging from the lack of pyruvate decarboxylation caused by thiamine deficiency (21-24) to the impairment of the electron transport chain caused by dysfunctional structure of the respiratory mitochondrial enzymes, induced, for example, by nitric oxide (25) or oxygen radicals (26). Another possible explanation for this association, although physiologically indistinguishable from the aforementioned mechanisms, entails the dysregulation of the microcirculation leading to peripheral shunting $(3,27)$.

\section{Lactate and Metabolic Acidosis}

An increase in the concentration of lactate results in metabolic acidosis (i.e., a process leading to an excess of negative strong ions) $(14,28)$. However, acidemia (i.e., an abnormally high proton concentration [low $\mathrm{pH}]$ ) is not necessarily present if other processes simultaneously promote a compensatory decrease in negative strong ions, with consequent widening of strong ion difference and restoration of $\mathrm{pH}$ toward normality. The kidney has a pivotal role in correcting for the excess of lactate. Indeed, given that $\mathrm{Pa}_{\mathrm{CO}_{2}}$ in our population was similar across lactate sextiles, the compensatory mechanisms when present were mainly caused by an offsetting increase in the strong ion difference by the kidney. 


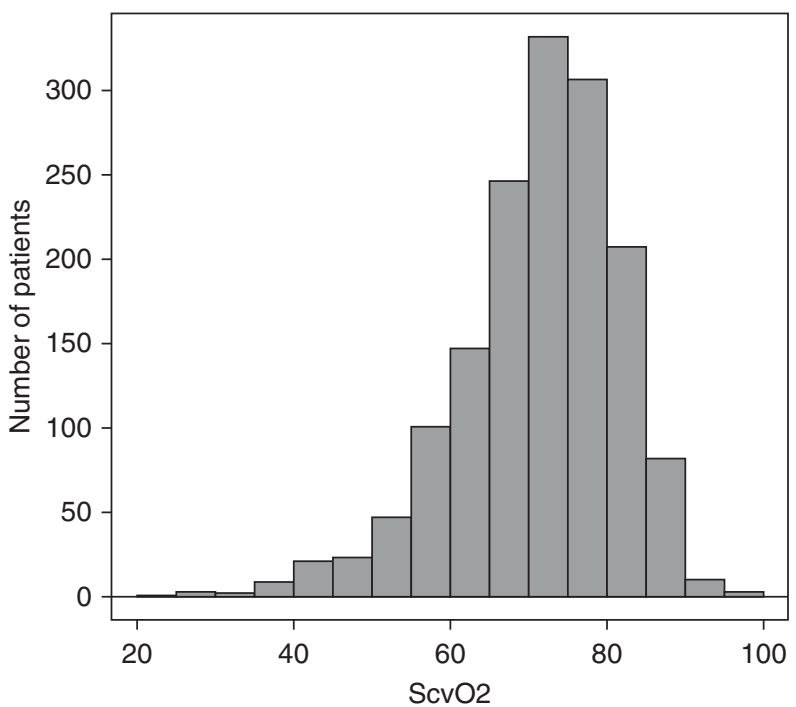

Figure 2. Observed frequency of distribution of central venous oxygen saturation $\left(\mathrm{Scv}_{\mathrm{O}_{2}}\right)$ at baseline measured in the whole population at ICU admission. As shown, only a minority of patients presented an $\mathrm{Scv}_{\mathrm{O}_{2}}$ consistent with oxygen transport deficit. Note, however, that the extreme values of $\mathrm{Scv}_{\mathrm{O}_{2}}$ (one patient $<25 \%$ and three patients $>95 \%$ ) are likely artifactual.

To better understand the relation between hyperlactatemia and acidemia, we have introduced the concept of alactic $\mathrm{BE}$, which helps to quickly discriminate between metabolic acidosis secondary to lactate from metabolic acidosis caused, for example, by an accumulation of fixed acids (unmeasured strong anions). The role of renal function on the acid-base balance in sepsis is explicitly quantified by the alactic BE.
The association of a negative alactic BE with creatinine $>2 \mathrm{mg} / \mathrm{dl}$ indicates that fixed acids other than lactate are retained in the plasma, meaning that the kidney is no longer able to compensate for the lactic acidosis because of an associated renal dysfunction. An alactic $\mathrm{BE}$ of zero (observed at creatinine $\sim 2 \mathrm{mg} / \mathrm{dl}$ ) suggests that the kidney is still able to clear fixed acids but cannot fully
A

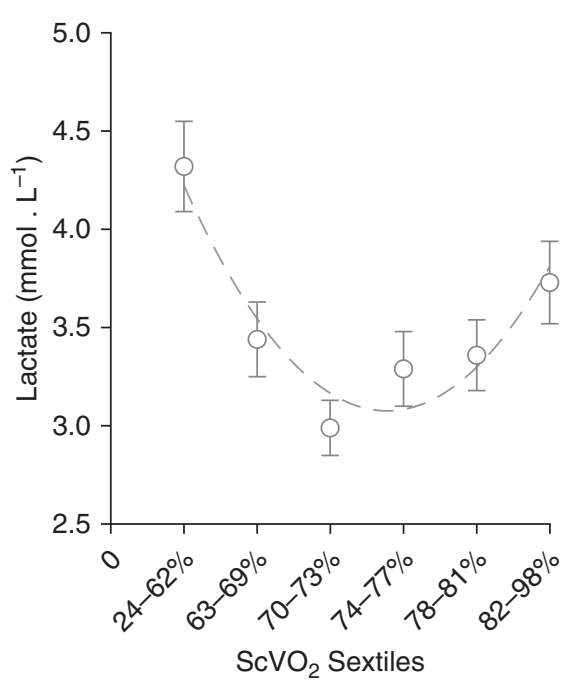

B

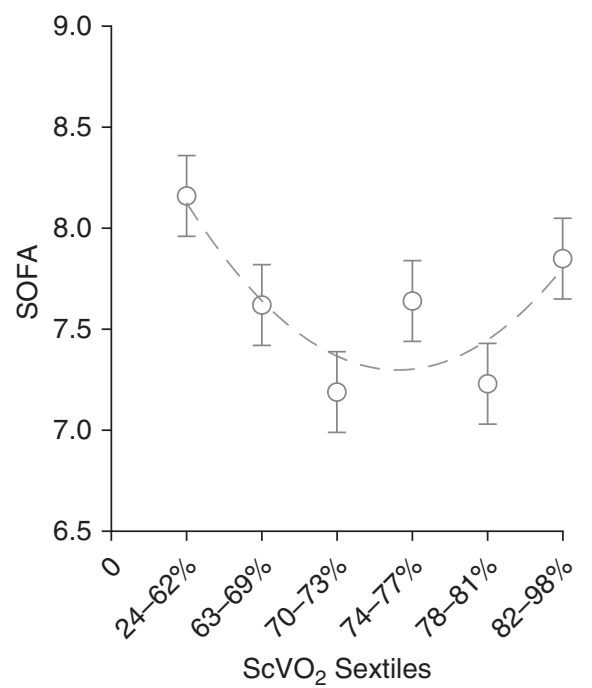

"compensate" for the acidosis induced by lactate.

A positive alactic $\mathrm{BE}$ (generally with a creatinine $<2 \mathrm{mg} / \mathrm{dl}$ ) suggests the presence of metabolic alkalosis, usually caused by diuretics or volume contraction (29). In summary, although an abnormally high lactate per se nearly always indicates acidosis of some severity, the degree of associated acidemia depends on renal ability to compensate. The concept of alactic BE is a simple, novel, and potentially useful method to immediately detect and track these phenomena over time. The classical $\mathrm{BE}$ includes all the information given by the alactic BE. However, alactic $\mathrm{BE}$ has more practical diagnostic and therapeutic potential. Indeed, a negative alactic $\mathrm{BE}$, observed in these patients with sepsis, alerts the physician to that fact that the renal function is impaired (unable to compensate for an excess of negative strong ions), whereas a positive alactic BE may indicate an additional process leading to metabolic alkalosis (e.g., excess use of diuretics and volume contraction). It should be noted that the alactic $\mathrm{BE}$ clearly differs from the anion gap [i.e., (sodium + potassium) (chloride + bicarbonate)], because this latter variable does not distinguish between lactate and other fixed acids. If the lactate is added to the anion gap computation, the alactic BE differs

\section{C}

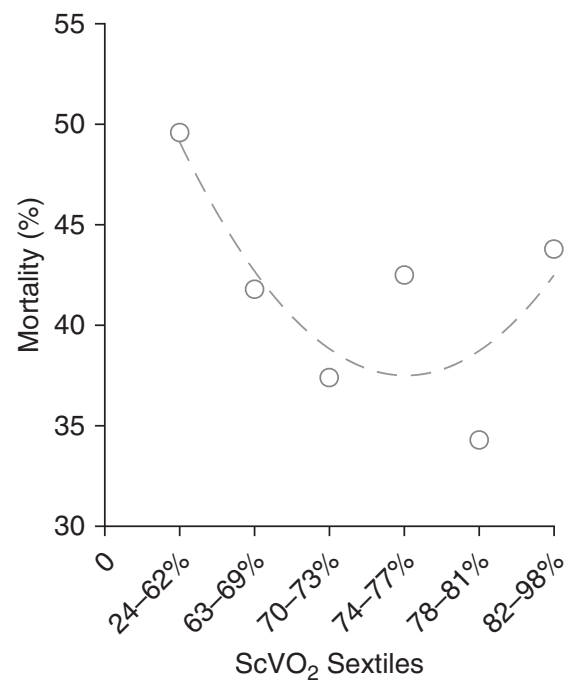

Figure 3. $(A-C)$ Lactate $(A)$, Sequential Organ Failure Assessment score $(B)$, and mortality $(C)$ as a function of central venous oxygen saturation sextiles at baseline (ICU admission). Data are presented as mean $\pm \mathrm{SE}$. Scv $\mathrm{O}_{2}=$ central venous oxygen saturation; SOFA = Sequential Organ Failure Assessment score. 
A

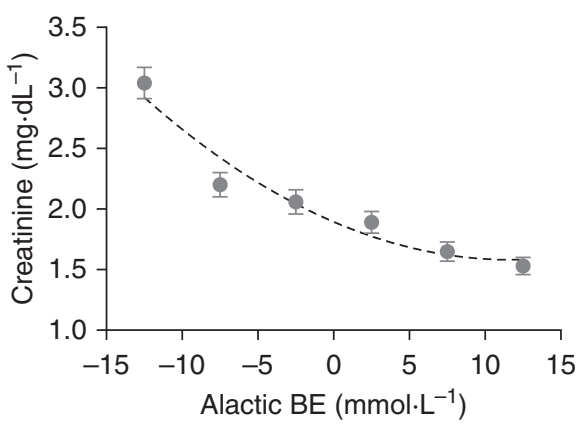

C

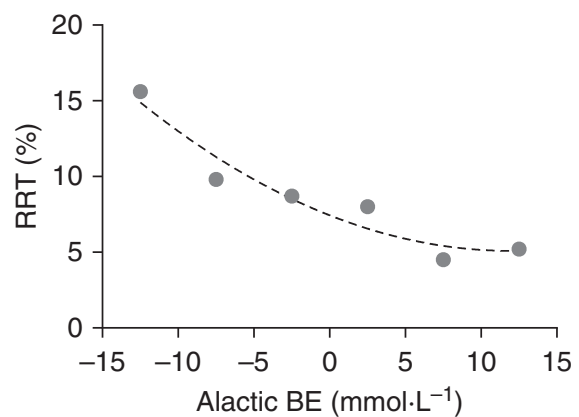

B

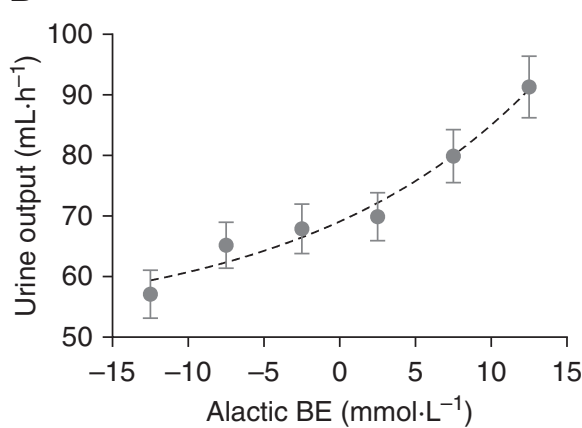

D

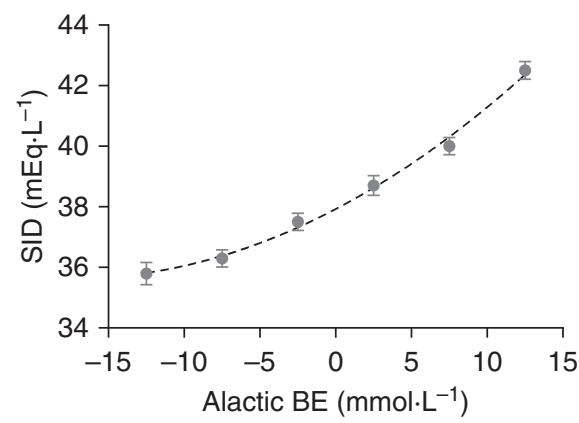

Figure 4. $(A-D)$ Creatinine $(A)$, diuresis $(B)$, renal-replacement therapy $(C)$, and simplified strong ion difference $\left[\left(\mathrm{Na}^{+}+\mathrm{K}^{+}\right)-\mathrm{Cl}^{-}\right](D)$ as a function of alactic base excess sextiles at baseline (ICU admission). Data are presented as mean $\pm \mathrm{SE}$. $\mathrm{BE}=$ base excess; $\mathrm{RRT}=$ renal-replacement therapy; $\mathrm{SID}=$ strong ion difference.

because it refers to a standard $\mathrm{PC}_{\mathrm{O}_{2}}$ and $\mathrm{pH}$, whereas the anion gap does not. The weak correlation between alactic $\mathrm{BE}$ and anion gap (adjusted $R^{2}=0.113$ ) is reported in Figure E6.

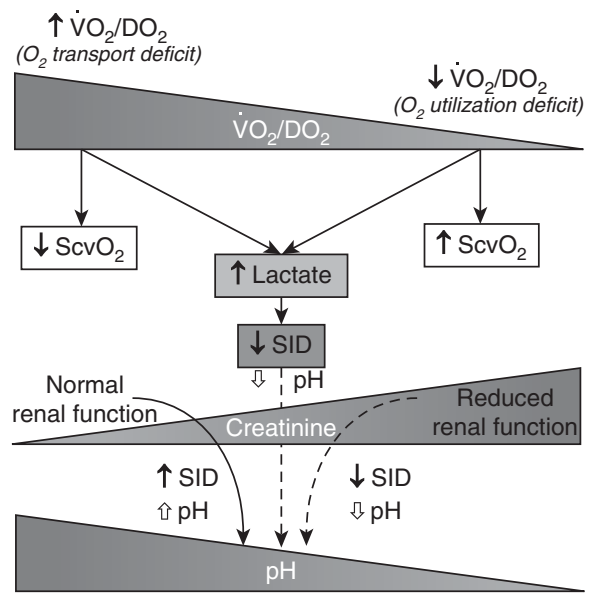

Figure 5. Lactate metabolic pathways and kidney response. The arrows' direction indicates increase $(\uparrow)$ or decrease $(\downarrow)$ of the given variable. See text and online supplement for further details. $\mathrm{Scv}_{\mathrm{O}_{2}}=$ central venous oxygen saturation; $\mathrm{SID}=$ strong ion difference.

\section{Possible Model of Sepsis Pathophysiology}

We believe that the controversial lactate shuttle theory (30), broadly applied in other settings of lactate generation, fits well with the bulk of our findings. That construct considers lactate the normal product of glycolysis (see online supplement for details and description). Indeed, the model emphasizes the importance of lactate as a central key of glucose metabolism, instead of considering it, as historically proposed (1), as the result of anaerobiosis. According to this model, all molecules of glucose entering the cytoplasm are metabolized into lactate, which is finally oxidized to $\mathrm{CO}_{2}$ and water. If lactate production exceeds oxidative capacity (e.g., excessive $\beta$-adrenergic stimulation, thiamine deficiency, respiratory chain impairment, lack of oxygen), excess lactate is transported out of the cells, usually in association with a proton (30). The decrease in $\mathrm{pH}$ caused by the increase in plasma lactate is sensed by the kidney, which decreases the urinary strong ion difference (31) to restore the normal plasma $\mathrm{pH}$.
The plasma concentration of lactate reaches a plateau if the rate of lactate production in the nonfunctioning metabolic units equals the rate of lactate oxidation by the metabolically active functioning units. Most organs, primarily the liver, may "clear" circulating lactate (i.e., completely oxidize lactate) and the rate of oxidation in the functioning metabolic units increases with the lactate input. Indeed, a strong relationship has been shown between exogenous lactate input and its oxidation in patients during dialysis (32), and the same phenomenon has been observed in experimental animal models (33).

Therefore, we may hypothesize that in sepsis, the lactate oxidation capability of the functioning metabolic units (see Figure E10) increases with the increased availability of lactate (34) (see Figures E8 and E11). Interestingly, other metabolites that normally are oxidized by the Krebs cycle within the mitochondria (e.g., nonesterified fatty acids) behave in sepsis as does lactate: increased levels promote higher rates of oxidation (35).

\section{Clinical Implications}

Our findings may help account for the ability of lactate to predict the severity and outcome of patients with sepsis. Indeed, we showed that whatever the prevalent mechanism underlying the deterioration in organ function in sepsis (i.e., impairment in the oxygen transport or oxygen use), the end result is an increase in the production of lactates and a decrease in their oxidation, leading to hyperlactatemia. However, despite this apparent similarity in outcome, a better understanding of the primary mechanism of hyperlactatemia, as we suggest in this model, might guide a more targeted and less indiscriminate approach to the management of sepsis. In strictly following the management guidance currently advocated, all patients with overt sepsis would receive similar amount of fluids, regardless of their mixed venous oxygen saturation (36).

Actually, a deficit in the oxygen transport, as suggested by low $\mathrm{Scv}_{\mathrm{O}_{2}}$, may justify a therapeutic approach aiming at increasing it, such as early goal-directed therapy (37), and, even better, correcting, if possible, the precise cause of oxygen transport impairment. In contrast, at high $\mathrm{Scv}_{\mathrm{O}_{2}}$ (impaired oxygen use) the same therapeutic approach may seem, at best, ineffective, as suggested by recent 
randomized controlled trials (18-20). We may rationally wonder whether, in such cases, the mandated use of a fixed amount of fluid has a sound pathophysiologic rationale, and whether this approach is devoid of adverse consequences, as suggested by studies reporting positive fluid balance, renal dysfunction, and worse outcome after aggressive fluid replacement in sepsis $(38,39)$.

We suggest that patients might be first stratified on the basis of $\mathrm{Scv}_{\mathrm{O}_{2}}$ to understand the origin of lactate production, and then on the basis of the alactic BE to better understand organ (i.e., kidney) perfusion and volemia. Changes in this simple parameter over time may facilitate early restoration of appropriate fluid balance and/or prompt the use of RRT.

\section{Conclusions}

Our results indicate that in patients with sepsis: 1) lactate is a powerful marker of illness severity; 2) abnormal lactate levels, in established sepsis, seem to be generated primarily by impaired oxygen transport in the minority of cases, whereas in the majority, high lactate more likely results from impaired tissue oxygen use; and 3) the degree of acidemia or alkalemia depends primarily on renal function. The alactic $\mathrm{BE}$ offers a potentially useful way to estimate renal capability of handling the disturbance to acid-base equilibrium. A clear recognition of the mechanisms underlying lactate elevation should result in an improved therapeutic approach for the individual, particularly regarding the aggressiveness of fluid administration.

Author disclosures are available with the text of this article at www.atsjournals.org.

Acknowledgment: The authors thank Dr. Peter Suter for his fruitful review and critical evaluation of the manuscript.

\section{References}

1. Broder G, Weil MH. Excess lactate: an index of reversibility of shock in human patients. Science 1964;143:1457-1459.

2. Brealey D, Brand M, Hargreaves I, Heales S, Land J, Smolenski R, et al. Association between mitochondrial dysfunction and severity and outcome of septic shock. Lancet 2002;360:219-223.

3. Ince C, Mik EG. Microcirculatory and mitochondrial hypoxia in sepsis, shock, and resuscitation. J Appl Physiol (1985) 2016;120:226-235.

4. Hamanaka RB, Chandel NS. Cell biology: Warburg effect and redox balance. Science 2011;334:1219-1220.

5. James JH, Luchette FA, McCarter FD, Fischer JE. Lactate is an unreliable indicator of tissue hypoxia in injury or sepsis. Lancet 1999; 354:505-508.

6. Valenza F, Aletti G, Fossali T, Chevallard G, Sacconi F, Irace M, et al. Lactate as a marker of energy failure in critically ill patients: hypothesis. Crit Care 2005;9:588-593.

7. Garcia-Alvarez M, Marik P, Bellomo R. Sepsis-associated hyperlactatemia. Crit Care 2014;18:503.

8. Caironi P, Tognoni G, Masson S, Fumagalli R, Pesenti A, Romero M, et al.; ALBIOS Study Investigators. Albumin replacement in patients with severe sepsis or septic shock. N Engl J Med 2014;370: 1412-1421.

9. Vincent JL, Moreno R, Takala J, Willatts S, De Mendonça A, Bruining H, et al. The SOFA (Sepsis-related Organ Failure Assessment) score to describe organ dysfunction/failure: on behalf of the working group on sepsis-related problems of the European Society of Intensive Care Medicine. Intensive Care Med 1996;22:707-710.

10. Le Gall JR, Lemeshow S, Saulnier F. A new Simplified Acute Physiology Score (SAPS II) based on a European/North American multicenter study. JAMA 1993;270:2957-2963.

11. Singer M, Deutschman CS, Seymour CW, Shankar-Hari M, Annane D, Bauer $\mathrm{M}$, et al. The third international consensus definitions for sepsis and septic shock (Sepsis-3). JAMA 2016;315:801-810.

12. Vasques F, Duscio E, Romitti F, Pasticci I, Caironi P, Meessen J, et al. Septic shock-3 vs 2: an analysis of the ALBIOS study. Crit Care 2018;22:237.

13. Berend K. Diagnostic use of base excess in acid-base disorders. $N$ Engl J Med 2018;378:1419-1428.

14. Kellum JA, Elbers PWG. Stewart's textbook of acid-base. Morrisville, NC: Lulu Press; 2009.

15. Reinhart K, Kuhn HJ, Hartog C, Bredle DL. Continuous central venous and pulmonary artery oxygen saturation monitoring in the critically ill. Intensive Care Med 2004;30:1572-1578.

16. Levy B. Lactate and shock state: the metabolic view. Curr Opin Crit Care 2006;12:315-321.

17. Alegría L, Vera M, Dreyse J, Castro R, Carpio D, Henriquez C, et al. A hypoperfusion context may aid to interpret hyperlactatemia in sepsis-3 septic shock patients: a proof-of-concept study. Ann Intensive Care 2017;7:29.
18. Peake SL, Delaney A, Bailey M, Bellomo R, Cameron PA, Cooper DJ, et al.; ARISE Investigators; ANZICS Clinical Trials Group. Goaldirected resuscitation for patients with early septic shock. $N$ Engl $J$ Med 2014;371:1496-1506.

19. Yealy DM, Kellum JA, Huang DT, Barnato AE, Weissfeld LA, Pike F, et al.; ProCESS Investigators. A randomized trial of protocolbased care for early septic shock. N Engl J Med 2014;370: 1683-1693.

20. Mouncey PR, Osborn TM, Power GS, Harrison DA, Sadique MZ, Grieve RD, et al.; ProMISe Trial Investigators. Trial of early, goaldirected resuscitation for septic shock. N Engl J Med 2015;372: 1301-1311.

21. Donnino MW, Carney E, Cocchi MN, Barbash I, Chase M, Joyce N, et al. Thiamine deficiency in critically ill patients with sepsis. J Crit Care 2010;25:576-581.

22. Marik PE, Khangoora V, Rivera R, Hooper MH, Catravas J. Hydrocortisone, vitamin $\mathrm{C}$, and thiamine for the treatment of severe sepsis and septic shock: a retrospective before-after study. Chest 2017;151:1229-1238.

23. Donnino MW, Andersen LW, Chase M, Berg KM, Tidswell M, Giberson $\mathrm{T}$, et al.; Center for Resuscitation Science Research Group. Randomized, double-blind, placebo-controlled trial of thiamine as a metabolic resuscitator in septic shock: a pilot study. Crit Care Med 2016;44:360-367.

24. Woolum JA, Abner EL, Kelly A, Thompson Bastin ML, Morris PE, Flannery $\mathrm{AH}$. Effect of thiamine administration on lactate clearance and mortality in patients with septic shock. Crit Care Med 2018;46: 1747-1752.

25. Brown GC. Regulation of mitochondrial respiration by nitric oxide inhibition of cytochrome c oxidase. Biochim Biophys Acta 2001; 1504:46-57.

26. Turrens JF. Mitochondrial formation of reactive oxygen species. $J$ Physiol 2003;552:335-344

27. De Backer D, Donadello K, Sakr Y, Ospina-Tascon G, Salgado D, Scolletta S, et al. Microcirculatory alterations in patients with severe sepsis: impact of time of assessment and relationship with outcome. Crit Care Med 2013:41:791-799.

28. Kishen R, Honoré PM, Jacobs R, Joannes-Boyau O, De Waele E, De Regt $\mathrm{J}$, et al. Facing acid-base disorders in the third millennium - the Stewart approach revisited. Int J Nephrol Renovasc Dis 2014;7: 209-217.

29. Rose BD. Clinical physiology of acid-base and electrolyte disorders. New York, NY: McGraw-Hill; 2001.

30. Brooks GA. The science and translation of lactate shuttle theory. Cell Metab 2018;27:757-785.

31. Gattinoni L, Carlesso E, Cadringher P, Caironi P. Strong ion difference in urine: new perspectives in acid-base assessment. Crit Care 2006; 10:137.

32. Wright DA, Forni LG, Carr P, Treacher DF, Hilton PJ. Use of continuous haemofiltration to assess the rate of lactate metabolism in acute renal failure. Clin Sci (Lond) 1996;90:507-510. 
33. Zanella A, Patroniti N, Isgrò S, Albertini M, Costanzi M, Pirrone F, et al. Blood acidification enhances carbon dioxide removal of membrane lung: an experimental study. Intensive Care Med 2009;35: 1484-1487.

34. Levy B, Gibot S, Franck P, Cravoisy A, Bollaert PE. Relation between muscle $\mathrm{Na}+\mathrm{K}+$ ATPase activity and raised lactate concentrations in septic shock: a prospective study. Lancet 2005;365:871-875.

35. Samra JS, Summers LK, Frayn KN. Sepsis and fat metabolism. Br J Surg 1996;83:1186-1196.

36. Rhodes A, Evans LE, Alhazzani W, Levy MM, Antonelli M, Ferrer R, et al. Surviving sepsis campaign: international guidelines for management of sepsis and septic shock: 2016. Crit Care Med 2017;45:486-552.
37. Rivers E, Nguyen B, Havstad S, Ressler J, Muzzin A, Knoblich B, et al.; Early Goal-Directed Therapy Collaborative Group. Early goaldirected therapy in the treatment of severe sepsis and septic shock. N Engl J Med 2001;345:1368-1377.

38. Payen D, de Pont AC, Sakr Y, Spies C, Reinhart K, Vincent JL; Sepsis Occurrence in Acutely III Patients (SOAP) Investigators. A positive fluid balance is associated with a worse outcome in patients with acute renal failure. Crit Care 2008;12:R74.

39. Maitland K, Kiguli S, Opoka RO, Engoru C, Olupot-Olupot P, Akech SO et al.; FEAST Trial Group. Mortality after fluid bolus in African children with severe infection. N Engl J Med 2011;364: 2483-2495. 